\title{
The Legend of Zelda and the Portrayal of Women in Gaming History
}

\author{
Norachase Prom ${ }^{1}$
}

${ }^{1}$ Lovejoy High School, Lucas, Texas, USA

\section{ABSTRACT}

This paper aims to analyze the change in the cultural depictions of female characters in video gaming history through the developments of the character Princess Zelda over the course of ten games from The Legend of Zelda Series, spanning from 1986 to 2017. The ten games were divided into three categories by the developers that managed the series, games by Shigeru Miyamoto (1986 - 1998), Takashi Tezuka (2002 - 2009), and Eiji Aonuma. (2008 - 2019), respectively. Under each developers' tenure, Princess Zelda's portrayal was altered to adapt the cultural view of women during their time. The results of this literary analysis was that Princess Zelda's constantly evolving depiction represents two events in the gaming industry: the transition from an eastern perspective to a western perspective regarding the view of women in the gaming industry, and the changing demographics in the video game market to more female consumers.

\section{Introduction}

According to American Historian Kelly Wenig from Iowa State University, at the height of the Cold War in the mid to late 1900s, video games were created to demonstrate the progress of technology on both sides of the conflict. Games such as Pong produced in the United States and Tetris from the Soviet Union featured simplistic graphics yet provided much entertainment for its consumers. The increased popularity of this new form of entertainment led to the rise of the video game industry. At the start of the 1980s, the industry mainly catered to male adolescents, as Nintendo producer Shigeru Miyamoto explained in a 2013 interview that "arcades [in the 1980's] were not places girls went into often. And so [gaming companies] didn't even consider making a character that would be playable for girls" (Kotaku). As gaming systems became more intricate, video games incorporated elements of story-telling in addition to more detailed graphics but still primarily appealed only to young boys. Earlier games such as Donkey Kong, Super Mario Bros, and Dragon's Lair had one thing in common: the "Damsel In Distress" trope. This is a common theme found in these games in which the player assumes the role of the male protagonist, and the goal of the game is to rescue or save the "damsel," a female character who acts as a "reward" or "trophy" for completing the game.

Among these games were The Legend of Zelda (LoZ) series from Nintendo, which adhered to many of the same storytelling conventions in its earlier releases. The series covers a long period of the history of video games, churning out a game almost every two years since 1986. LoZ, like many video games made in the late 1980s, appealed to a mostly male demographic, with many of its plot lines revolving around a male protagonist going through a number of trials and obstacles in order to rescue a female character, Zelda. As Nintendo's hardware capabilities became more complex, the writers behind $L o Z$ felt that they were no longer restrained by limitations imposed by past systems, according to a 2001 interview with series director Shigeru Miyamoto. One way the writers of the game tried to make the story more complex is to make changes to the series's supporting character, Princess Zelda, who, as a result of this change is often advertised by Nintendo's executives and writers themselves as one of gaming's "most evolved" characters; however, some inspiration behind many of Zelda's character developments comes from real-world events that subsequently impacted the gaming community and industry. 
For a brief overview of the series, Nintendo created $L o Z$ in 1986 under the action-adventure genre in a medieval-fantasy setting. The game is set in the fictional kingdom of Hyrule and features multiple reincarnations of three main characters: Link, the playable character, Princess Zelda (Figure 1), the damsel in distress, and Ganondorf, the series' main antagonist. In many of these games, Princess Zelda is trapped, imprisoned, or held hostage by Ganondorf and rescued by Link. However, as newer versions of these games were released, this pattern of storytelling in the Zelda series became less prevalent.

\section{Literature Review}

\section{Princess Zelda and The Legend of Zelda series}

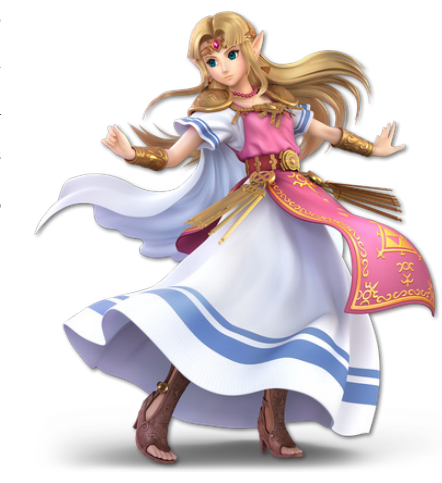

Figure 1

The most expansive analysis regarding Princess Zelda's character is a 2019 paper published by Melissa Huntley and Wind Goodfriend, psychologists at Buena Vista University. The two authors argue that in all games, Princess Zelda fits, to varying degrees, a common trope: The Damsel in Distress. However, despite Princess Zelda consistently locked into the role of being rescued, Huntley and Goodfriend summarized that throughout the games, Zelda's involvement in the story gets bigger. However, the two researchers only observed Zelda's role as a female character, and not any connections to real-world events or outside developments.

There have also been multiple in-depth analyses of Zelda among individual games; one was by researcher Clair McBride, who argues in her 2019 paper that Princess Zelda's role in The Legend of Zelda: Ocarina of Time (1998) serves as her struggle for independence and freedom, and that she is only respected by other characters when under a male disguise.

\section{Female protagonists in narrative games}

In the past, there was various research on female video game characters; such examples include papers by Psychologist Esther Maccalum-Stewart, and papers by researcher Katherine Roberts. Female characters in earlier video games were heavily analyzed by other researchers as well, being the first playable female characters in gaming, and many gaming developers would use these earlier characters as a framework for female protagonists in future games. What both Maccalum-Stewart and Roberts found was that, for early female video game characters, the intentions behind their designs were subject to fetishization, but over time, these characters gradually undergo redesigns that were less sexualized and more emphasis was placed on personality traits.

There was also an extensive study on female protagonists in 2016 by Mimi Perreault, and other Doctors of Philosophy from different universities. Perrault et al. discuss the popularity of games with female characters as the leading role. They have also noted that in the early 2010s there was a trend in the number of female characters having a dominant role in narrative video games, and those characters are represented in a diverse range of character depictions, making them popular among female players.

\section{Women in Video Games}

A 2019 paper by Olatz Lopez-Fernandez and other psychologists at Nottingham Trent University investigates the role of women in gaming culture, both before and during the 21st century. Lopez-Fernandez Et Al stated that early on, Video games have been a strictly male dominated hobby but as time progresses, it has opened up to a female audience. 
In a section of their paper titled Women's Position in Gaming Culture, they summarized that in the industry "the competence of women in [high ranking] positions is often questioned" as well as their interests in gaming.

Another research by Lopez Fernandez discussed how, as the number of female gamers increase, game developers have attempted methods to cater to that demographic, usually through marketing, or with the contents of the games themselves.

\section{Focus of Inquiry}

This paper aims to analyze Princess Zelda's gradual change from a two-dimensional damsel in distress to a more dynamic character, to connect the series of changes to events that led to increasingly progressive female representation, and analyze how those events led to the decisions for Zelda's characterization. It is hypothesized that since the LoZ series is a Japanese game, and Japan is a conservative culture regarding their views toward women, Zelda's change is mostly influenced by developments in the western hemisphere. This paper aims to find that a microcosm of issues on women's fight for equality are represented in a series of video games.

\section{Method}

This research was conducted through a literary analysis of the games in the Legend of Zelda series; however, only mainline games that include Princess Zelda as a character were analyzed. Spinoff games and non-gaming media such as comics and the television series will not be analyzed because these media are either not produced by Nintendo, contain non-canonical information, or contradict Zelda's character in the game. That leaves ten playable games, with publish dates ranging from 1986 to 2017. In the ten games, various aspects of Princess Zelda, including her physical appearance in official art, interactions with other characters (especially Link), and her actions in cutscenes will be observed to note the changes made to Princess Zelda's portrayal in its narrative context.

The ten games were grouped into three categories by development teams, games directed by Shigeru Miyamoto (1986 - 1998), Takashi Tezuka (2002 - 2009), and Eiji Aonuma. (2008 - 2019), respectively. The reason behind this categorization was that each of these developers had different approaches to Zelda's depiction allowing for a general idea of how Princess Zelda was revised under each head developer. Afterward, for each game, any notable change in Zelda's depictions such as appearance and Zelda's traits will be identified and, for the years that each of the games were released, a development regarding women in the gaming industry occurring in either Japan or North America from that same year will be paired with the game. This is done so that any change by the developers on Zelda's character would be connected to a real-world event in the gaming industry to discern whether they may have influenced the developer's decision.

The process of documenting the data for each of the ten games are as follows: under the names of each of the three different developers, a timeline by order of tenure was created, the ten games were sorted chronologically as bullet points under their respective developers to indicate which games are developed by which producer. Next, for each of the individual games, a brief plot summary, retrieved by viewing in-game cutscenes, regarding Zelda is provided and then compared, with any and all statements from interviews, developer's notes or documents (see Literature Review above) detailing the decisions behind Zelda's depiction or character motivations or developer motivations. Finally, any real-world event regarding women in video game culture using the time frame immediately before or after a game was developed, allows for possible motivation for changes to the plot structure or characterization. The reasoning behind the use of bullet points laid chronologically is that they are effective in comparing the plot and characterization to the developer's notes to the real-world events. 


\title{
Results and Analysis
}

\author{
Shigeru Miyamoto (1986-1998)
}

In a 1993 interview with American author, David Sheff, Shigeru Miyamoto explained that he took his initial inspiration for the franchise from his experiences as a young boy traveling the wilderness in the outskirts of Kyoto, which to Miyamoto at the time, meant the ' unknown.' This experience of curiosity became the core design philosophy of the franchise in terms of both gameplay and story. Miyamoto's original intentions for The Legend of Zelda was that it was meant to serve as a "coming of age" game for the adolescent male demographic because he wanted to convey that same feeling of adventure he had experienced as a boy. For the plot of the story, Miyamoto wanted saving a princess from captivity to be the main goal of the game, stating that it alludes to "the idea that [young boys] want to be seen as the 'knight in shining armour.'” Miyamoto's reasoning behind choosing Zelda as the princess's name was from Zelda Fitzgerald, wife of famous author F. Scott Fitzgerald, who Miyamoto viewed as a "fair maiden" and later based Zelda's personality off her.

Seven games have been written under the direction of Shigeru Miyamoto, though only four of those have Zelda as a character: The Legend of Zelda (1986), The Legend of Zelda II: The Adventures of Link (1987), The Legend of Zelda: A Link to the Past (1991) and The Legend of Zelda: Ocarina of Time (1998). The first three games follow one basic structure: Link has to defeat an evil entity in order to rescue Princess Zelda and save the kingdom of Hyrule, the game's setting, from impending doom. Miyamoto had similar formats of writing with other games he had directed at the time, such as Super Mario Bros and Kid Icarus, two games that involve a male playable character fighting monsters to rescue a female damsel in distress, most of the time being royalty.

The first two games, The Legend of Zelda, and The Legend of Zelda II: Adventures of Link, were the start of Miyamoto's experimentation with narrative video games. In a 2016 interview with gaming journalist Stephen Totilo, Miyamoto explained that Zelda's inclusion as a female character contrasts an otherwise male-centric narrative of the two games. In these games, Zelda is represented as a two-dimensional sprite with simple plain colors and has limited voice lines (Figure 2), only appearing at the end. In these games, Link has no relations with Zelda, only rescuing the princess as a "prize" for defeating the main antagonist, Ganondorf.

In 1991, during the development of The Legend of Zelda: A Link to the Past, Miyamoto

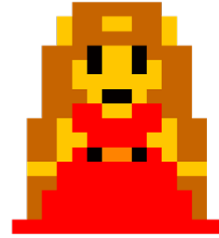

Figure 2 expressed in his 2016 interview that when asking for feedback from many of his fans, North American consumers had difficulty distinguishing between Princess Zelda and Princess Peach from Miyamoto's Super Mario series. To set Zelda apart from Peach, Miyamoto had given her magical powers such as telepathy and a "wise" personality, as he calls it, which set the precedent for future depictions of Zelda. In A Link to the Past, Zelda is held hostage in her castle's dungeons. She uses telepathy to contact Link, to rescue her. Link then stealthily escorts Zelda out of the castle. Unlike the previous two games, there appears to be a large age difference between Zelda and Link, while in the Japanese version of the game, it is implied that Link is in his early teens while Zelda is around the age of 20 (Figure 3). Furthermore, the overseas version only mentions that Zelda is older than Link. The decision to alter Zelda's age by Miyamoto can be interpreted as an effort to make the character of Princess Zelda appear to have prevailing wisdom due to her age and maturity in comparison to Link; in Japanese culture, older members of society are perceived to be wise, According to a 2011 anthropological study on Japanese customs regarding the elderly by Miyumi Karasawa and other cultural psychologists from various universities. 
The Legend of Zelda: Ocarina of Time (OT) presents a drastic shift to Miyamoto's storytelling pattern for the series. With Nintendo's 1996 console, the N64 and its three-dimensional graphics, Miyamoto stated in a 2001 interview that he wanted a "cinematic" game which the players could interact with the game's environment, and he chose $L o Z$ for a sequel for two reasons: a game with a medieval fantasy setting would be visually appealing, and to better illustrate the theme of "coming of age" for adolescent boys. His ambitious story was split into two acts set seven years apart. In the first act, Princess Zelda is tasked as the kingdom's soothsayer at the age of ten. Growing suspicious of the King's advisor and the game's soon-to-be antagonist, Ganondorf, Zelda tasks Link to retrieve artifacts she believes Ganondorf is after. Eventually, the Kingdom is overthrown by Ganondorf, and Ganondorf hexes Link into a seven-year slumber and pursues the Princess for her magical powers. In the second act, Link awakens in a ruined Hyrule and is tasked with Zelda's rescue. Throughout his journey, Link is aided by a man named Sheik, who teaches Link combative skills. In the end, it is revealed that Sheik is Zelda in disguise (Figure 4), as she needed a plan to hide from Ganondorf. However, just moments after Sheik is revealed to be Zelda, she is captured by Ganondorf and is rescued by the end of the game.

In scenes where Zelda is disguised as Sheik, She expresses to Link that she is free to roam the world and become an adventurer, know-

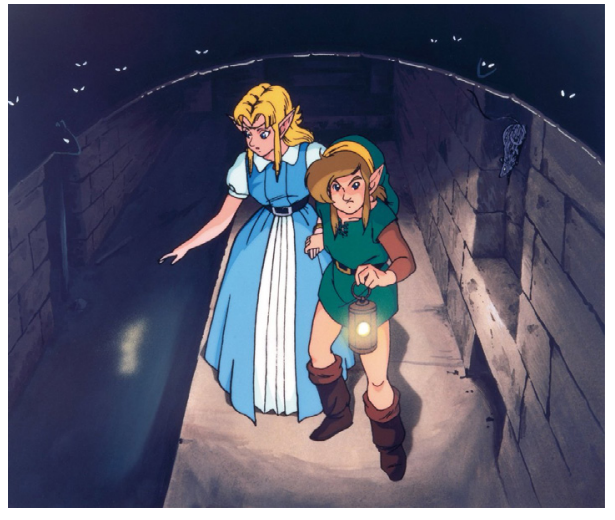

Figure 3 ing that she can only do this under a male alter-ego. Through her disguise, Zelda forms an intimate bond with Link, a relationship that her father forbade due to Link's status as a commoner. However, the freedom Zelda enjoys as Sheik is lost once she reveals her true identity and is captured by Ganondorf. After her rescue, Zelda is pressured to act as a ruler of the kingdom and reverts back to fulfilling her feminine duties as a princess, despite the absence of her father's influence, even refusing to maintain her friendship with Link, as he is a commoner. Media critic and womens' rights activist Anita Sarkessian argues that this characterization of Zelda serves as an example of how female video game characters have limited roles in early video games, and that they are only able to enjoy an equal footing with male characters when disguised as men.

\section{Takashi Tezuka (2002-2009)}

After the production of the Ocarina of Time, Miyamoto was assigned projects outside the LoZ franchise, so he entrusted its management to Takashi Tezuka. Unlike Miyamoto, Tezuka wanted his writing team to be more democratic when it came to the game's writing decisions. Regarding the relationship between Link and Princess Zelda, in a 2017 interview with gaming journalist Tom Phillips, Tezuka's writing team
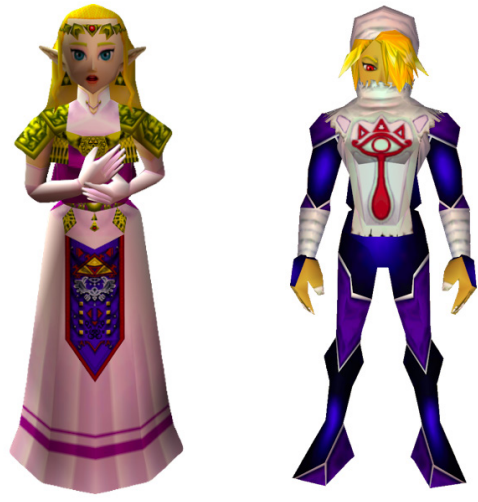
believed that portraying the princess as a damsel could potentially feel "out of touch" with its western audience, but they also wanted their relationship to stand out from other western narrative games at the time. According to a cross-cultural analysis on Nintendo by researchers Connor Garret and Ostin Kurniawan, many executives at Nintendo during the late 2000s believed that as a Japanese company, they are obliged to express japanese societal values more freely to foriegn markets.

Figure 4 
Likely a part of this change in the company's philosophy, the art of the series adopted a more stylized Japanese design under Tezuka's management, according to an interview with Nintendo President Iwata, this change in art style was to give the series "a distinct Japanese flair" (Figure 5). Tezuka's writing team believed that Link and Zelda's relationship should model that of the ideal couple that represented Japanese values, which in the case of LoZ, meant that both the men and the women share equal responsibilities, yet the man is a more dominant partner in the relationship, while it is the woman's duty to provide council, according to a study on Japanese gender roles by Melinda Belarmino and Melinda Roberts, experts on gender studies at the University of Southern Indiana.

In his career, Tezuka produced three games: The Legend of Zelda: Wind Waker (2002), The Legend of Zelda: Phantom Hourglass (2007), and The Legend of Zelda: Spirit Tracks (2009); all three are connected story-wise. Tezuka's writing philosophy is represented well in these games, where Link and Zelda often fought alongside each other against the antagonist; however, these games show that it is obvious Link is the stronger and dominant one

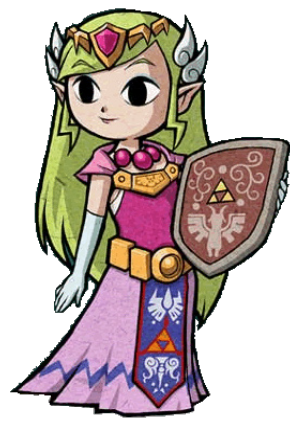

Figure 5 in this relationship, while Zelda is depicted as a wise-but-physically-weak figure who guides Link.. While their idea of an equal relationship seemed good on paper, based on an interview with Nintendo's thenpresident, Satoru Iwata, he explained that many fans, especially those outside of Japan, felt that this iteration of Zelda constituted a negative portrayal of her character and demanded a more positive depiction.

In The Legend of Zelda: Wind Waker, (WW) the game takes place in a Hyrule that is in a flooded state, leaving a series of Archipelagos. In this game, Zelda is cursed with memory loss, oblivious to the fact that she is a princess. She believes her name is Tetra (Figure 6), becomes a pirate captain-the only female in the crew. She meets

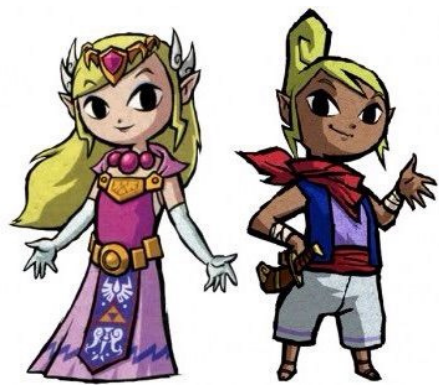

Figure 6 Link in a hostile encounter but agrees to aid Link under the condition that he help her find the "royal treasure." Throughout the game, Tetra and her crew fight alongside Link and It is revealed that the treasure is Zelda's memories, and her pirate crew are members of the royal family who also lost their memories. At the end of the game, Zelda rejects her identity and continues to live as Tetra, going on adventures with Link and her crew as pirates. This is the first game in which Princess Zelda is shown fighting alongside Link, not through magical powers, but with a physical weapon, being the bow.

This iteration of Zelda is a sharp contrast to her previous counterparts in that she is depicted in a tomboyish personality, and often partakes in physical altercations. It had also served as a contrast to her previous iteration in $O T$, whereas OT Zelda was unable to break free from the expectations placed upon her as a princess, WW Zelda/Tetra chose to ignore her responsibilities, instead living the rest of her life as her alter ego.

Five years later, Tezuka created a sequel to $W W$ and called it The Legend of Zelda: Phantom Hourglass. Set immediately after $W W$, Link and Tetra embark on other adventures; however, at the start of the game, as the crew boards a haunted ship, Tetra is trapped in the ship and bound by its curse. Tetra is not seen again until the end of the game when Link manages to break the curse. While researchers Huntley and Goodfriend lauded Tetra's depiction in $W W$ as a positive portrayal of a female character, they also believed that Tetra's character development was reduced to a simple damsel again due to her role in $P H$, as they argued that up until $W W$, there was a gradual buildup for Zelda's dynamic role, only for it to be ignored or forgotten in $\mathrm{PH}$.

Tezuka's games were commercially successful in the western market due to his unique take on the series' gameplay mechanics but had become marketable during a time when female representation in the gaming industry was being questioned. According to cyberculture expert Torill E. Mortensen from the IT University of Copenhagen, the late 2000s and early 2010s saw the \#GamerGate controversy, in which prominent women in the gaming industry addressed the sexism in the industry and how that sexism stems from the depictions of women in video games. In 2008, one activist, Anita Sarkessian, used $L o Z$ as an example of why there is a need for progressivism in the female 
representation. Likely as a response, Tezuka concluded his trilogy with The Legend of Zelda: Spirit Tracks (2009), which addresses several criticisms by having Zelda's soul accompany Link as he tries to reunite her with her body. This constitutes a compromise between the series' traditional structure and the demand for active female roles.

\section{Eiji Aounuma (2009-2017)}

Lastly, the series's third and current developer, Eiji Aounuma, worked from 2009 to 2017. Unlike Tezuka, Aonuma acted as the sole decision maker in terms of writing the story, and as a response to the growing fan demand for a better depiction of Zelda's character, In an interview with Matt Peckham of Time Magazine, Aonuma hired many female staff in his writing and art design team in order to have an input on how a female character should be better portrayed. In that same interview, Aonuma stated that he was well aware of the broad appeal of the LoZ series, and noted that many Zelda games made by him had a higher ratio of female gamers compared to past games in the series made by Miyamoto or Tezuka.

During Aonuma's time on the LoZ team, Nintendo developed a new goal of differentiating their intellectual properties; specifically, the goal was to address the monotonous designs of Nintendo's female characters, based on the interview Aounuma had with Peckham. This decision was reflected in Aonuma's first game, The Legend of Zelda: Twilight Princess (TP) (2008), although during this time, Tezuka was overseeing two Zelda games, TP and Spirit Tracks, he assigned Aounuma as the story director for the former, while still under his management.

In a 2008 interview with the Famitsu Column, a Japanese gaming magazine, Aonuma wanted to prioritize the "distress" aspect as much as the "damsel" part of Zelda's character. According to Aonuma, one way he altered

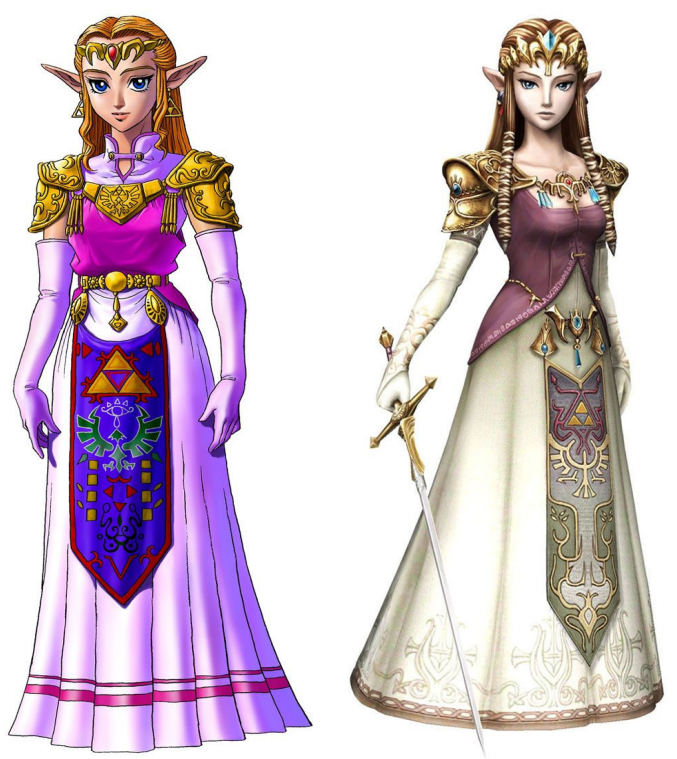

Figure 7 Zelda's personality was to give her a somber and depressed personality while emphasizing her role as a princess by portraying her speech and actions in a regal manner. When visually distinguishing her from previous counterparts, Aonuma wanted to show signs of distress by giving her more dark and muted colors while affirming her "damsel" role by adorning her dress with jewelry and intricate patterns (Figure 7).

In that same interview with the Famitsu Column, Aonuma also wanted to give an additional role to Zelda. In TP, Princess Zelda acts as the new political ruler in Hyrule after her father's recent death, the first time she is depicted in this manner. She also acts as an army commander to defend the kingdom from a foriegn invasion. When her army is defeated, Zelda is captured as a prisoner of war. Humiliated, Zelda uses her telepathic powers to contact a peasant farmer, Link, to infiltrate the castle and rescue her. Upon meeting Link, Zelda assures him that his "divine duty" is to only aid and protect Zelda, not to form a relationship.

In later games, Aonuma placed Link's adventures to rescue and help Zelda as a backdrop to Zelda's character arc, often focusing on what she does in captivity. This decision is clearly shown in his first debut as a director in The Legend of Zelda: Skyward Sword (SS) (2011). In this game, in order to earn her title as princess, Zelda is required to undergo service at a military academy. Throughout the story, Zelda faced hardships in the academy as the only female. To redeem herself, Zelda embarks on a scouting mission in enemy territory, only to be captured. A fellow student named Link is tasked to save the princess, but throughout Link's journey, the player is shown cutscenes of Zelda in captivity, in which she hears degrading comments 
from her captors and scenes where Zelda uses her military knowledge to escape and fight. In the end, Zelda eventually becomes a princess.

Another pattern Aonuma broke with Zelda's character design in the series is that in every game he produced, Princess Zelda is burdened with the responsibilities of a princess. In games by Miyamoto or Tezuka, Zelda accepts the restrictions placed on her as part of her duty of being princess, including being captured. Aonuma's games present Zelda as a person who struggles to uphold her responsibilities. However, in his 2017 installment, The Legend of Zelda: Breath of the Wild (BotW), Zelda rejects those restrictions. In BotW, Zelda develops an interest in scholarly pursuits, which in its narrative context, is only reserved for men. She rejects a life of being constrained to her royal duties and as a result of this, Zelda's father, the King, appoints Link as Zelda's personal bodyguard to not only protect her, but to ensure that Zelda is fulfilling her duties. Zelda views Link as the embodiment of those restrictions and resents him.

Both $S S$ and BotW were released during a period when, as the International Game Developers association (IGDA) stated, the gaming industry saw an increase in the employment of female staff as well as the number of women in high ranking positions in the industry. An outspoken critic of the $L o Z$ series, Anita Sarkeesian, wrote in her 2017 article that Zelda's depictions in both games, though more emphasis on BotW, was a "progressive step in the right direction for [Japanese] representation of women in [the gaming industry], as Zelda is now put "in equal footing as her male counterpart, Link".

In a 2019 interview with Matt Peckham of Time Magazine, Aonuma explained that there was one primary factor behind his decisions of Zelda's characters, particularly her dynamic characterization in BotW: one is a trend in western games that places more women in protagonist roles, a trend similar to what Mimi Perreault et al. had described. Aonuma alluded to a recent development that "changed the way [gaming industries] view women," referring to Japanese gaming companies cracking down on sexually explicit content regarding the depiction of women in April 2019, according to Takashi Mochizuki of the Wall Street Journal. Mochizuki stated that this crackdown was part of a series of events in response to the \#MeToo movement, originating in the U.S. In that same article Female industry leaders called for a more "healthy, realistic, and positive" depiction of women in addition to more inclusion of female staff. This movement was aimed towards Japanese developers, Nintendo included, which traditionally had a more conservative approach on women's representation and were urged to address these concerns.

Nintendo's decision to adapt their games to reflect the social standing of women in the early 2010s coincided with the change in the video gaming market from Japan to Europe and North America as well as more gaming companies being founded outside of Japan. Economist Guiddita de Prato from the Institute for Prospective Technologies at Seville stated that in the 1980s and early 1990s, Japan was the leading video game producer as well as the largest video game market, but at the start of the 2000s, gaming companies in Europe and North America gained influence. At the turn of the century, North American and European demand for video games increased as noted by economist Casey O'Donnell.

Meanwhile, according to a survey conducted by the International game developer's association, roughly 36 percent of all gamers were women in 2017, which they claimed was twice the amount ten years ago. As a response to this growing population of female gamers, Japanese gaming companies such as Nintendo hired female employees, according to an interview with Nintendo executives by Forbes magazine. Female employees are especially important in the marketing department, as they help the advertisements for the games appeal to potential female consumers.

As for the future, former president of Nintendo of America, Reggie Fils-Aime, believes that Japanese gaming companies will cooperate with both its western markets. He believes it will foster a cultural exchange between the two regions. As for $L o Z$, it is reported that Eiji Aonuma will continue the trend of Princess Zelda's positive portrayal. As of June 2019, Nintendo announced that they intend to depict Zelda with short hair and not wearing a dress, a deviation from her typical feminine design. The details for this upcoming game are yet to be known, but Author Kevin Binversie states in his 2019 paper that he expects that the constant change in Zelda's portrayal will not be going away anytime soon. At the same month, an article by Forbes discusses the future impacts of the \#MeToo movement in the gaming industry. In the article, they argue that because there are already women now holding prominent positions in the industry, that goal can be realized in the future. 


\section{References}

Barder, Ollie. "Nintendo Responds To Questions About Women In Game Development In Japan” Forbes. 10 Feb. 2019. 27 Sep. 2019.

Belarmino, Melanie and Melinda R. Roberts. "Japanese Gender Role Expectations and Attitudes: A Qualitative Analysis of Gender Inequality” Bridgewater State University. Aug. 2019. 23 Apr. 2020.

Binversie, Kevin. "Zelda Is Gaming's Most Evolved Female Character” Washington Examiner. 1 May. 2017. 30 Sep. 2019.

Bunting, Geoffrey. "Nintendo's Worrying Treatment of Women and Femininity” Medium. 17 Sep. 2017. 1 Oct. 2019.

De Prato, Giudiita. Et Al. "Innovations in the Video Game Industry: Changing Global Markets" Institute for Prospective Technologies, European Commission. Print. 2014. 12 Jan. 2020.

Garret, Connor and Ostin Kurniawan. "Nintendo Cross-Cultural Analysis: United States and Japan" Web. Dec. 2018. 12 Jan 2020.

Handlen, Zack. "Princess Zelda's clumsy evolution reflects a series in search of its soul" AV. 18 Feb. 2016. 3 Oct. 2019.

Huntly, Melissa and Wind Goodfriend. "The Legend Herself: From Damsel In Distress To Princess Of Power" Print. 2019. 3 Oct. 2019.

Janz, Joerenz and Raynel G. Martis. "The Lara Phenomenon: Powerful Female Characters in Video Games" Sex roles Journal of reaserch. 2 Feb. 2007. 18 Oct. 2019.

Karasawa, Mayumi. Et Al. "Cultural Perspectives on Aging and Well-Being: A Comparison of Japan and the U.S." National Center for Biotechnology Information. 30 Sep. 2011. 14 Nov. 2019.

Lopez-Fernandez, Olatz. Et Al. "Female Gaming, Gaming Addiction, and the Role of Women Within Gaming Culture: A Narrative Literature Review" Frontiers in Psychiatry. 10 Jul. 2019. 2 Feb. 2020.

MacCallum-Stewart, Esther.“'Take That, Bitches!' Refiguring Lara Croft in Feminist Game Narratives” The University of the West of England. 2014. Print. 18 Oct. 2019.

McBride, Clare. " The Legend of Zelda: Ocarina of Time is about Zelda Becoming an adult too" SyFywire. 30 Mar. 2019. 4 Oct. 2019.O’Donnell, Casey. “The North American Game Industry”. 2013. Print. 22 Feb. 2020.

Mochizuki, Takashi. "Sony Cracks Down on Sexually Explicit Content in Games" Wall Street Journal. 16 Apr. 2019. 2 Oct. 2019.

$\mathrm{Ng}$, Raymond and Robb Lindgren. "Examining the effects of avatar customization and narrative on engagement and learning in video games" Print. 13 Oct. 2013. 17 Nov. 2019. 
Peckham, Matt. "Next Link May Not Be a Girl, But He’s Androgynous by Design” Time Magezine. 15 Jun. 2016. 25 Sep. 2019.

Perreault, Mimi. Et Al. "Depictions of Female Protagonists in Digital Games: A Narrative Analysis of 2013 DICE Award-Winning Digital Games” Print. Nov. 2016. 17 Nov. 2019.

Philips, Tom. "The big Zelda: Breath of the Wild interview” Eurogamer. 19 Jan. 2017. 4 Oct. 2019.

Roberts, Katherine. "A sociological exploration of a female character in the Metroid videogames series" The Computer Games Journal. 10 May. 2017. 30 Sep. 2019.

Schott, Gareth and Thomas Sioban. “The Impact of Nintendo's 'For Men’ Advertising Campaign on a Potential Female Market” Eludamos. Journal for Computer Game Culture. 2008. 25 Sep. 2019.

Sheff, David. "Game Over: How Nintendo Zapped an American Industry, Captured Your Dollars, and Enslaved Your Children” 1993. Book. 2 Oct. 2019.

“Subdued Ceremony” Nintendo. 2017. Author’s screenshot.

Totilo, Stephen. “Shigeru Miyamoto and the Damsel in Distress” Kotaku. 20 Jun. 2013. 3 Oct. 2019.

Wenig, Kelly. "Fighting the Cold War in the Virtual Streets: Video Games as a Source Base for Social and Cultural History" Iowa State University. Sep. 2011. 17 Nov. 2019.

Yee, Nick. "Just How Important Are Female Protagonists?” Quantic Foundry. 29 Aug. 2017. 30 Sep. 2019.

"Zelda.” Smashbros. 2018. Web. 23 Feb. 2020. 